\title{
Correction to: The Seifert-Van Kampen Theorem
}

\section{Correction to:}

\section{Chapter 12 in: C. Bray et al., Algebraic Topology,}

https://doi.org/10.1007/978-3-030-70608-1_12

The original version of the chapter was inadvertently published without incorporating the author's corrections in Theorem 12.4, which have now been corrected as follows: In the first equation, the ldots have been changed to cdots. In the second equation, the cdots have been changed to ldots. The book and the chapter have been updated with the changes. 